\title{
Accelerated life testing design using geometric process for pareto distribution
}

\author{
Mustafa Kamal*, Shazia Zarrin, Arif-Ul-Islam \\ Department of Statistics \& Operations Research, Aligarh Muslim University, Aligarh, India \\ *Corresponding author E-mail: kamal19252003@gmail.com
}

\begin{abstract}
In many of the studies concerning Accelerated life testing (ALT), the log linear function between life and stress which is just a simple re-parameterization of the original parameter of the life distribution is used to obtain the estimates of original parameters but from the statistical point of view, it is preferable to work with the original parameters instead of developing inferences for the parameters of the log-linear link function. In this paper the geometric process is used for the analysis of accelerated life testing under constant stress for Pareto Distribution. Assuming that the lifetimes under increasing stress levels form a geometric process, estimates of the parameters are obtained by using the maximum likelihood method for complete data. In addition, asymptotic interval estimates of the parameters of the distribution using Fisher information matrix are also obtained. The statistical properties of the parameters and the confidence intervals are illustrated by a Simulation study.
\end{abstract}

Keywords: Maximum Likelihood Estimation; Reliability Function; Fisher Information Matrix; Asymptotic Confidence Interval; Simulation Study.

\section{Introduction}

Due to the global competition for the development of new products in a short time and to achieve customer's satisfaction manufacturing industries continuously improving their manufacturing design which makes today's products and materials highly reliable. Since, in life testing experiments, time-to-failure data is used to quantify the product's failure-time distribution and its associated parameters under normal operating conditions, Therefore, Testing under normal operating conditions require a very long period of time and need an extensive number of units under test. So it is usually costly and impractical to perform reliability testing under normal conditions. Under these circumstances Accelerated life testing (ALT) is the best choice to test the products. ALT is a quick way to obtain information about the life distribution of a material, component or product in which products are tested at higher than usual level of stress, which yields shorter life but, hopefully, do not change the failure mechanisms. Three types of stress loadings are usually applied in accelerated life tests: constant stress, step stress and linearly increasing stress. The constant stress loading, which is a time-independent test setting, has several advantages over the time-dependent stress loadings. For example, most products are assumed to operate at a constant stress under normal use. Therefore, a constant stress test mimics actual use. Failure data obtained from ALT can be divided into two categories: complete (all failure data are available) or censored (some of failure data are missing). Complete data consist of the exact failure time of test units, which means that the failure time of each sample unit is observed or known. In many cases when life data are analyzed, all units in the sample may not fail. This type of data is called censored or incomplete data.

Constant stress ALT with different type of data and planning has been studied by many authors. For example, Yang [1] proposed an optimal design of 4-level constant-stress ALT plans considering different censoring times. Pan et al. [2] proposed a bivariate constant stress accelerated degradation test model by assuming that the copula parameter is a function of the stress level that can be described by a logistic function. Chen et al. [3] discuss the optimal design of multiple stress constant accelerated life test plan on non-rectangle test region. Watkins and John [4] considers constant stress accelerated life tests based on Weibull distributions with constant shape and a log-linear link between scale and the stress factor which is terminated by a Type II censoring regime at one of the stress levels. Fan and Yu [5] discuss the reliability analysis of the constant stress accelerated life tests when a parameter in the generalized gamma lifetime distribution is linear in the stress level. Ding et al. [6] dealt with Weibull distribution to obtain accelerated life test sampling plans under type I progressive interval censoring with random removals. Ahmad et al. [7], Islam and Ahmad 
[8], Ahmad and Islam [9], Ahmad, et al. [10] and Ahmad [11] discuss the optimal constant stress accelerated life test designs under periodic inspection and Type-I censoring.

The concept of geometric process is introduced by Lam [12], in repair replacement problems. Since then a large amount of studies in maintenance problems and system reliability have been shown that a geometric process model is a good and simple model for analysis of data with a single trend or multiple trends. For example Lam and Zhang [13] apply the geometric process model in the analysis of a two-component series system with one repairman. Lam [14] studied the geometric process model for a multistate system and determined an optimal replacement policy to minimize the long run average cost per unit time. Zhang [15] used the geometrical process to model a simple repairable system with delayed repair. So far, there are only three studies that utilize the geometric process in the analysis of accelerated life test. Huang [16] introduced the GP model for the analysis of ALT with complete and censored exponential samples under the constant stress. Kamal et al. [17] extended the GP model for the analysis of ALT with complete Weibull failure data under constant stress. Zhou et al. [18] implemented the Geometric Process in the constant stress accelerated life test model based on the progressive Type-I hybrid censored Rayleigh failure data.

In this paper, the use of geometric process model is considered in the analysis of constant stress accelerated life testing for the Pareto distribution with complete data. Maximum likelihood estimates of parameters and their asymptotic confidence intervals are obtained. The performance of the estimates is evaluated by a simulation study.

\section{The model and test procedure}

\subsection{The geometric process}

A geometric process describes a stochastic process $\left\{X_{n}, n=1,2, \ldots\right\}$, where there exists a real valued $\lambda>0$ such that $\left\{\lambda^{n-1} X_{n}, n=1,2, \ldots\right\}$ forms a renewal process. The positive number $\lambda>0$ is called the ratio of the GP. It is clear to see that a GP is stochastically increasing if $0<\lambda<1$ and stochastically decreasing if $\lambda>1$. Therefore, the GP is a natural approach to analyze data from a series of events with trend.

It can be shown that if $\left\{X_{n}, n=1,2, \ldots\right\}$ is a GP and the pdf of $X_{1}$ is $f(x)$ with mean $\mu$ and variance $\sigma^{2}$ then the pdf of $X_{n}$ will be given be $\lambda^{n-1} f\left(\lambda^{n-1} x\right)$ with $E\left(X_{n}\right)=\mu / \lambda^{n-1}$ and $\operatorname{Var}\left(X_{n}\right)=\sigma^{2} / \lambda^{2(n-1)}$. Thus $\lambda, \mu$ and $\sigma^{2}$ are three important parameters of a GP.

\subsection{The pareto distribution}

The probability density function, cumulative distribution function and survival function of the Pareto distribution with scale parameter $\theta$ and shape parameter $\alpha$ are given respectively by

$$
\begin{array}{ll}
f(x)=\frac{\alpha \theta^{\alpha}}{(\theta+x)^{\alpha+1}} ; & x>0, \theta>0, \alpha>0 \\
F(x)=1-\frac{\theta^{\alpha}}{(\theta+x)^{\alpha+1}} ; & x>0, \theta>0, \alpha>0 \\
S(x)=\frac{\theta^{\alpha}}{(\theta+x)^{\alpha+1}} &
\end{array}
$$

\subsection{Assumptions and test procedure}

I. Suppose that an accelerated life test with $s$ increasing stress levels in which a random sample of $n$ identical items is placed under each stress level and start to operate at the same time. Let $x_{k i}, i=1,2, \ldots, n, k=0,1,2, \ldots, s$ denote observed failure time of $i^{\text {th }}$ test item under $k^{\text {th }}$ stress level. Whenever an item fails, it will be removed from the test and test is continue till all the test items failed (complete data) and the exact failure time $x_{k i}$ of item is observed.

II. The product life follows a two parameter Pareto distribution given by (1) at any stress.

III. The shape parameter $\alpha$ is constant, i.e. independent of stress.

IV. The scale parameter is a $\log$-linear function of stress. That is, $\log \theta_{i}=a+b S_{i}$, where $a$ and $b$ are unknown parameters depending on the nature of the product and the test method. 
V. Let random variables $X_{0}, X_{1}, X_{2}, \ldots, X_{s}$, denote the lifetimes under each stress level, where $X_{0}$ denotes item's lifetime under the design stress at which items will operate ordinarily and sequence $\left\{X_{k}, k=0,1,2, \ldots, s\right\}$ forms a geometric process with ratio $\lambda>0$.

The assumption (V) which will be used in this study may be stronger than the commonly used Assumptions (I-IV) in usual discussion of ALTs in literature without increasing the complexity of calculations. The next theorem discusses how the assumption of geometric process (assumption V) is satisfied when there is a log linear relationship between a life characteristic and the stress level (assumption IV).

Theorem 2.1: If the stress level in an ALT is increasing with a constant difference then the lifetimes under each stress level forms a Geometric Process. That is, If $S_{k+1}-S_{k}$ is constant for $k=1,2, \ldots, s-1$, then $\left\{X_{k}, k=0,1,2, \ldots, s\right\}$ forms a Geometric Process. Or Log Linear and GP model are equivalent when the stress increases arithmetically.

Proof: From assumption (IV), it can easily be shown that

$\log \left(\frac{\theta_{k+1}}{\theta_{k}}\right)=b\left(S_{k+1}-S_{k}\right)=b \Delta S$

Now (2) can be rewritten as

$\frac{\theta_{k+1}}{\theta_{k}}=e^{b \Delta S}$

This shows that the increased stress levels form an arithmetic sequence with a constant difference $\Delta S$. Now assume that

$\frac{\theta_{k+1}}{\theta_{k}}=\frac{1}{\lambda}$

It is clear from (3) that

$\theta_{k}=\frac{1}{\lambda} \theta_{k-1}=\frac{1}{\lambda^{2}} \theta_{k-2}=\ldots=\frac{1}{\lambda^{k}} \theta$

The PDF of the product lifetime under the $k^{\text {th }}$ stress level is

$$
\begin{aligned}
f_{X_{k}}(x) & =\frac{\alpha \theta_{k}{ }^{\alpha}}{\left(\theta_{k}+x\right)^{\alpha+1}} \\
& =\frac{\alpha\left\{\frac{1}{\lambda^{k}} \theta\right\}^{\alpha}}{\left(\frac{1}{\lambda^{k}} \theta+x\right)^{\alpha+1}} \\
& =\lambda^{k} \frac{\alpha \theta^{\alpha}}{\left(\theta+\lambda^{k} x\right)^{\alpha+1}}
\end{aligned}
$$

This implies that

$f_{X_{k}}(x)=\lambda^{k} f_{X_{0}}\left(\lambda^{k} x\right)$

Now, the definition of GP and (4) have the evidence that, if density function of $X_{0}$ is $f_{X_{0}}(x)$, then the probability density function of $X_{k}$ will be given by $\lambda^{k} f\left(\lambda^{k} x\right), k=0,1,2, \cdots, s$. Therefore, it is clear that lifetimes under a sequence of arithmetically increasing stress levels form a geometric process with ratio $\lambda$.

Therefore the probability density function of a test item at the $k^{\text {th }}$ stress level by using theorem 2.1 can be written by

$f_{X_{k}}(x \mid \alpha, \theta, \lambda)=\frac{\lambda^{k} \alpha \theta^{\alpha}}{\left(\theta+\lambda^{k} x\right)^{\alpha+1}}$ 


\section{The maximum likelihood method of estimation}

Here the maximum likelihood method of estimation is used because ML method is very robust and gives the estimates of parameter with good statistical properties. In this method, the estimates of parameters are those values which maximize the sampling distribution of data. However, ML estimation method is very simple for one parameter distributions but its implementation in ALT is mathematically more intense and, generally, estimates of parameters do not exist in closed form, therefore, numerical techniques such as Newton Method and some computer programs are used to compute them.

The likelihood function using geometric process for the Pareto distribution under constant stress accelerated life testing for complete data is given by

$L=\prod_{k=1}^{s} \prod_{i=1}^{n} \frac{\lambda^{k} \alpha \theta^{\alpha}}{\left(\theta+\lambda^{k} x_{k i}\right)^{\alpha+1}}$

The log-likelihood function corresponding (5) can be rewritten as

$l=\sum_{k=1}^{s} \sum_{i=1}^{n}\left[k \ln \lambda+\ln \alpha+\alpha \ln \theta-(\alpha+1) \ln \left(\theta+\lambda^{k} x_{k i}\right)\right]$

MLEs of $\alpha, \theta$ and $\lambda$ are obtained by solving the equations $\frac{\partial l}{\partial \alpha}=0, \frac{\partial l}{\partial \theta}=0$ and $\frac{\partial l}{\partial \lambda}=0$, where

$\frac{\partial l}{\partial \alpha}=\frac{n s}{\alpha}+n s \ln \theta-\sum_{k=1}^{s} \sum_{i=1}^{n} \ln \left(\theta+\lambda^{k} x_{k i}\right)$

$\frac{\partial l}{\partial \theta}=\frac{n s \alpha}{\theta}-(\alpha+1) \sum_{k=1}^{s} \sum_{i=1}^{n}\left[\frac{1}{\left(\theta+\lambda^{k} x_{k i}\right)}\right]$

$\frac{\partial l}{\partial \lambda}=\sum_{k=1}^{s} \sum_{i=1}^{n}\left[\frac{k}{\lambda}-\frac{(\alpha+1) k \lambda^{k-1} x_{k i}}{\left(\theta+\lambda^{k} x_{k i}\right)}\right]$

Obviously, it is difficult to obtain a closed form solution to nonlinear equations (7), (8) and (9). So, Newton-Raphson method is used to solve these equations simultaneously to obtain $\hat{\alpha}, \hat{\theta}$ and $\hat{\lambda}$.

\section{Asymptotic confidence interval estimates}

According to large sample theory, the maximum likelihood estimators, under some appropriate regularity conditions, are consistent and normally distributed. Since ML estimates of parameters are not in closed form, therefore, it is impossible to obtain the exact confidence intervals, so asymptotic confidence intervals based on the asymptotic normal distribution of ML estimators instead of exact confidence intervals are obtained here.

The Fisher-information matrix composed of the negative second partial derivatives of log likelihood function can be written as

$$
F=\left[\begin{array}{ccc}
-\frac{\partial^{2} l}{\partial \alpha^{2}} & -\frac{\partial^{2} l}{\partial \alpha \partial \theta} & -\frac{\partial^{2} l}{\partial \alpha \partial \lambda} \\
-\frac{\partial^{2} l}{\partial \theta \partial \alpha} & -\frac{\partial^{2} l}{\partial \theta^{2}} & -\frac{\partial^{2} l}{\partial \theta \partial \lambda} \\
-\frac{\partial^{2} l}{\partial \lambda \partial \alpha} & -\frac{\partial^{2} l}{\partial \lambda \partial \theta} & -\frac{\partial^{2} l}{\partial \lambda^{2}}
\end{array}\right]
$$

Elements of Fisher Information matrix are 


$$
\begin{aligned}
& \frac{\partial^{2} l}{\partial \alpha^{2}}=-\frac{n s}{\alpha^{2}} \\
& \frac{\partial^{2} l}{\partial \theta^{2}}=-\frac{n s \alpha}{\theta^{2}}+(\alpha+1) \sum_{k=1}^{s} \sum_{i=1}^{n} \frac{1}{\left(\theta+\lambda^{k} x_{k i}\right)^{2}} \\
& \frac{\partial^{2} l}{\partial \lambda^{2}}=-\sum_{k=1}^{s} \sum_{i=1}^{n} \frac{k}{\lambda^{2}}-(\alpha+1) \sum_{k=1}^{s} \sum_{i=1}^{n} k(k-1) \frac{\lambda^{k-2} x_{k i}}{\left(\theta+\lambda^{k} x_{k i}\right)} \\
& +(\alpha+1) \sum_{k=1}^{s} \sum_{i=1}^{n} k^{2} \frac{\lambda^{2 k-2} x_{k i}^{2}}{\left(\theta+\lambda^{k} x_{k i}\right)^{2}} \\
& \frac{\partial^{2} l}{\partial \alpha \partial \theta}=\frac{\partial^{2} l}{\partial \theta \partial \alpha}=\frac{n s}{\theta}-\sum_{k=1}^{s} \sum_{i=1}^{n} \frac{1}{\left(\theta+\lambda^{k} x_{k i}\right)} \\
& \frac{\partial^{2} l}{\partial \alpha \partial \lambda}=\frac{\partial^{2} l}{\partial \lambda \partial \alpha}=-\sum_{k=1}^{s} \sum_{i=1}^{n} k^{2} \frac{\lambda^{k-1} x_{k i}}{\left(\theta+\lambda^{k} x_{k i}\right)} \\
& \frac{\partial^{2} l}{\partial \theta \partial \lambda}=(\alpha+1) \sum_{k=1}^{s} \sum_{i=1}^{n} \frac{\lambda^{k-1} x_{k i}}{\left(\theta+\lambda^{k} x_{k i}\right)^{2}}
\end{aligned}
$$

Now, the variance covariance matrix can be written as

$$
\Sigma=\left[\begin{array}{ccc}
-\frac{\partial^{2} l}{\partial \alpha^{2}} & -\frac{\partial^{2} l}{\partial \alpha \partial \theta} & -\frac{\partial^{2} l}{\partial \alpha \partial \lambda} \\
-\frac{\partial^{2} l}{\partial \theta \partial \alpha} & -\frac{\partial^{2} l}{\partial \theta^{2}} & -\frac{\partial^{2} l}{\partial \theta \partial \lambda} \\
-\frac{\partial^{2} l}{\partial \lambda \partial \alpha} & -\frac{\partial^{2} l}{\partial \lambda \partial \theta} & -\frac{\partial^{2} l}{\partial \lambda^{2}}
\end{array}\right]^{-1}=\left[\begin{array}{ccc}
A \operatorname{Var}(\hat{\alpha}) & A \operatorname{Cov}(\hat{\alpha} \hat{\theta}) & A \operatorname{Cov}(\hat{\alpha} \hat{\lambda}) \\
A \operatorname{Cov}(\hat{\theta} \hat{\alpha}) & A \operatorname{Var}(\hat{\theta}) & A \operatorname{Cov}(\hat{\theta} \hat{\lambda}) \\
A \operatorname{Cov}(\hat{\lambda} \hat{\alpha}) & A \operatorname{Cov}(\hat{\lambda} \hat{\theta}) & A \operatorname{Var}(\hat{\lambda})
\end{array}\right]
$$

The $100(1-\gamma) \%$ asymptotic confidence interval for $\theta, \alpha$ and $\lambda$ are then given respectively as

$$
\left[\hat{\theta} \pm Z_{1-\frac{\gamma}{2}} \sqrt{A \operatorname{Var}(\hat{\theta})}\right],\left[\hat{\alpha} \pm Z_{1-\frac{\gamma}{2}} \sqrt{A \operatorname{Var}(\hat{\alpha})}\right] \text { and }\left[\hat{\lambda} \pm Z_{1-\frac{\gamma}{2}} \sqrt{A \operatorname{Var}(\hat{\lambda})}\right]
$$

\section{Simulation study}

The performance of the estimates can be evaluated through some measures of accuracy which are the standard error (SE), the mean squared error (MSE) and the coverage rate of asymptotic confidence intervals for different sample sizes and stress levels.

To conduct a simulation study to evaluate the performance of the methods for complete data, first a sample $x_{k i}, \quad k=1,2, \ldots, s, \quad i=1,2, \ldots, n$ is generated from Pareto distribution. The values of the parameters and number of stress levels are chosen to be $\theta=0.5, \alpha=1.5, \lambda=1.02$ and $s=3,5$. For different sample sizes $n=20,40, \ldots, 100$ and stress levels, ML estimates, the asymptotic standard error (SE), the mean squared error (MSE), lower and upper asymptotic CI limits and the coverage rate of the $95 \%$ confidence interval of parameters based on 400 simulations are obtained by our proposed model and summarized in Table 1 and 2. 
Table1: Simulations results based on complete data from GP Pareto with $\lambda=1.02, \alpha=1.5, \theta=0.5$ and $s=4$

\begin{tabular}{|c|c|c|c|c|c|c|c|}
\hline $\begin{array}{c}\text { Sample } \\
\text { Size } n\end{array}$ & Parameter & MLE & SE & MSE & LCL & UCL & $\begin{array}{c}\text { 95\% } \\
\text { Asymptotic } \\
\text { CI } \\
\text { Coverage }\end{array}$ \\
\hline & $\lambda$ & 1.0117 & 0.0102 & 0.0103 & 0.76482 & 1.1161 & 0.9361 \\
\hline 20 & $\alpha$ & 1.6331 & 0.2127 & 0.2250 & 0.8257 & 2.5853 & 0.9680 \\
\hline & $\theta$ & 0.5443 & 0.0241 & 0.0250 & 0.2752 & 0.8617 & 0.9680 \\
\hline & $\lambda$ & 1.0271 & 0.0052 & 0.0051 & 0.9169 & 1.2164 & 0.9795 \\
\hline 40 & $\alpha$ & 1.5206 & 0.0889 & 0.0738 & 0.8358 & 1.8764 & 0.9591 \\
\hline & $\theta$ & 0.5068 & 0.0098 & 0.0082 & 0.2786 & 0.6254 & 0.9591 \\
\hline & $\lambda$ & 1.0292 & 0.0035 & 0.0028 & 0.9449 & 1.1945 & 0.9795 \\
\hline 60 & $\alpha$ & 1.5027 & 0.0573 & 0.0432 & 0.9280 & 1.7933 & 0.9693 \\
\hline & $\theta$ & 0.5009 & 0.0064 & 0.0048 & 0.3093 & 0.5977 & 0.9693 \\
\hline 80 & $\lambda$ & 1.0199 & 0.0025 & 0.0024 & 0.9116 & 1.1029 & 0.9700 \\
\hline & $\alpha$ & 1.5218 & 0.0438 & 0.0367 & 1.1301 & 1.7545 & 0.9510 \\
\hline & $\theta$ & 0.5072 & 0.0048 & 0.0040 & 0.3767 & 0.6433 & 0.9500 \\
\hline 100 & $\lambda$ & 1.0192 & 0.0020 & 0.0018 & 0.9655 & 1.1516 & 0.9700 \\
\hline & $\alpha$ & 1.5197 & 0.0351 & 0.0329 & 1.0676 & 1.7441 & 0.9640 \\
\hline & $\theta$ & 0.5065 & 0.0039 & 0.0036 & 0.3558 & 0.5813 & 0.9600 \\
\hline
\end{tabular}

Table2: Simulations results based on complete data from GP Pareto with $\lambda=1.02, \alpha=1.5, \theta=0.5$ and $s=6$

\begin{tabular}{|c|c|c|c|c|c|c|c|}
\hline $\begin{array}{c}\text { Sample } \\
\text { Size } n\end{array}$ & Parameter & MLE & SE & MSE & LCL & UCL & $\begin{array}{c}\text { 95\% } \\
\text { Asymptotic } \\
\text { CI } \\
\text { Coverage }\end{array}$ \\
\hline & $\lambda$ & 1.0150 & 0.0029 & 0.0028 & 0.9318 & 1.1566 & 0.9587 \\
\hline 20 & $\alpha$ & 1.5693 & 0.1093 & 0.1035 & 0.8005 & 1.9462 & 0.9588 \\
\hline & $\theta$ & 0.5179 & 0.0132 & 0.0139 & 0.2668 & 0.6487 & 0.9587 \\
\hline & $\lambda$ & 1.0244 & 0.0015 & 0.0011 & 0.9676 & 1.1231 & 0.9898 \\
\hline 40 & $\alpha$ & 1.5068 & 0.0501 & 0.0353 & 0.9990 & 1.8124 & 0.9790 \\
\hline & $\theta$ & 0.5022 & 0.0057 & 0.0039 & 0.3330 & 0.6041 & 0.9797 \\
\hline & $\lambda$ & 1.0221 & 0.0011 & 0.0012 & 0.9520 & 1.0860 & 0.9400 \\
\hline 60 & $\alpha$ & 1.5163 & 0.0403 & 0.0373 & 1.1236 & 1.8982 & 0.9750 \\
\hline & $\theta$ & 0.5054 & 0.0044 & 0.0041 & 0.3745 & 0.6327 & 0.9730 \\
\hline & $\lambda$ & 1.0182 & 0.0008 & 0.0008 & 0.9928 & 1.1128 & 0.9700 \\
\hline 80 & $\alpha$ & 1.5227 & 0.0290 & 0.0290 & 1.0869 & 1.7042 & 0.9720 \\
\hline & $\theta$ & 0.5075 & 0.0032 & 0.0032 & 0.3623 & 0.5680 & 0.9732 \\
\hline & $\lambda$ & 1.0178 & 0.0006 & 0.0005 & 0.9636 & 1.0604 & 0.9600 \\
\hline 100 & $\alpha$ & 1.5175 & 0.0223 & 0.0194 & 1.2456 & 1.8200 & 0.9810 \\
\hline & $\theta$ & 0.5058 & 0.0024 & 0.0021 & 0.4152 & 0.6066 & 0.9800 \\
\hline
\end{tabular}

\section{Discussion and conclusions}

This paper deals with use of GP model in the analysis of constant stress ALT plan for Pareto distribution with complete data. The MLEs, SEs and MSEs of the model parameters were obtained. Based on the asymptotic normality, the coverage rate of $95 \%$ confidence intervals of the model parameters were also obtained.

Results in Table 1 and 2 indicate that $\hat{\theta}, \hat{\alpha}$ and $\hat{\lambda}$ estimates the true parameters $\theta, \alpha$ and $\lambda$ quite well with relatively small mean squared errors. For fixed $\theta, \alpha$ and $\lambda$ we find that as $n$ and $s$ increases, the mean squared errors and the asymptotic standard error (SE) of $\theta, \alpha$ and $\lambda$ decreases. It is also notice that the coverage probabilities of the asymptotic confidence interval are close to the nominal level and do not change much as sample size increases. From these results It may be concluded that the present model work well under complete data. 


\section{Acknowledgements}

This work has been funded by University Grants Commission under DRS-I (SAP) (Code: 1206).

\section{References}

[1] Yang, G. B., "Optimum constant-stress accelerated life-test plans, IEEE Transactions on Reliability", vol. 43, no. 4, (1994), pp. 575-581, available online: http://ieeexplore.ieee.org/stamp/stamp.jsp?tp=\&arnumber=370223\&isnumber=8488

[2] Pan, Z., N. Balakrishnan, and Quan Sun, "Bivariate constant-stress accelerated degradation model and inference", Communications in Statistics-Simulation and Computation, vol. 40, no. 2, (2011), 247-257, available online: http://dx.doi.org/10.1080/03610918.2010.534227

[3] Chen, W., Gao, L., Liu, J., Qian, P. and Pan, J., "Optimal design of multiple stress constant accelerated life test plan on non-rectangle test region", Chinese Journal of Mechanical Engineering, vol. 25, no. 6, (2012), pp. 1231-1237, available online: http://dx.doi.org/10.3901/CJME.2012.06.1231

[4] Watkins, A.J. and John, A.M., "On constant stress accelerated life tests terminated by Type II censoring at one of the stress levels", Journal of Statistical Planning and Inference, vol. 138, no. 3, (2008), pp 768-786, available online: http://dx.doi.org/10.1016/j.bbr.2011.03.031

[5] Fan, T. H. and Yu, C. H., "Statistical Inference on Constant Stress Accelerated Life Tests under Generalized Gamma Lifetime Distributions", Quality and Reliability Engineering International, (2012), available online: http://dx.doi.org/10.1002/qre.1412

[6] Ding, C., Yang, C. and Tse, S. K., "Accelerated life test sampling plans for the Weibull distribution under type I progressive interval censoring with random removals", Journal of Statistical Computation and Simulation, vol. 80, no. 8, (2010), pp. 903-914, available online: http://www.tandfonline.com/doi/abs/10.1080/00949650902834478

[7] Ahmad, N. Islam, A., Kumar, R. and Tuteja, R. K., "Optimal Design of Accelerated Life Test Plans Under Periodic Inspection and Type I Censoring: The Case of Rayleigh Failure Law", South African Statistical Journal, 28, (1994), pp. 27-35.

[8] Islam, A. and Ahmad, N., "Optimal design of accelerated life tests for the Weibull distribution under periodic inspection and type I censoring", Microelectronics Reliability, vol. 34, no. 9, (1994), pp. 1459-1468, available online: http://dx.doi.org/10.1016/0026-2714(94)90453-7

[9] Ahmad, N. and Islam, A., "Optimal accelerated life test designs for Burr type XII distributions under periodic inspection and type I censoring", Naval Research Logistics, vol. 43, (1996), pp. 1049-1077, available online: http://dx.doi.org/10.1002/(SICI)15206750(199612)43:8<1049::AID-NAV2>3.0.CO;2-E

[10] Ahmad, N., Islam, A. and Salam, A., "Analysis of optimal accelerated life test plans for periodic inspection: The case of exponentiated Weibull failure model", International Journal of Quality \& Reliability Management, vol. 23, no. 8, (2006), pp. 1019-1046, available online: http://dx.doi.org/ 10.1108/02656710610688194

[11] Ahmad, N., "Designing Accelerated Life Tests for Generalized Exponential Distribution with Log-linear Model", International Journal of Reliability and Safety, vol. 4, no. 2/3, (2010), pp. 238-264, available online: http://dx.doi.org/10.1504/IJRS.2010.032447

[12] Lam, Y., "Geometric process and replacement problem, Acta Mathematicae Applicatae Sinica", vol. 4, no. 4, (1988), pp. 366-377, available online: http://dx.doi.org/10.1007/BF02007241

[13] Lam, Y. and Zhang, Y. L., "Analysis of a two-component series system with a geometric process model”, Naval Research Logistics, vol. 43, no. 4, (1996), pp. 491-502, available online: http://dx.doi.org/10.1002/(SICI)1520-6750(199606)43:4<491::AID-NAV3>3.0.CO;2-2

[14] Lam, Y., "A monotone process maintenance model for a multistate system", Journal of Applied Probability, vol. 42, no. 1, (2005), pp. 1-14, available online: http://www.jstor.org/stable/30040765

[15] Zhang, Y. L. (2008): A geometrical process repair model for a repairable system with delayed repair, Computers and Mathematics with Applications, vol. 55, no. 8, (2008), pp. 1629-1643, available online: http://dx.doi.org/10.1016/j.camwa.2007.06.020

[16] Huang, S., "Statistical inference in accelerated life testing with geometric process model”, Master's thesis, San Diego State University, (2011), available online: http://hdl.handle.net/10211.10/1105

[17] Kamal, M., Zarrin, S., Saxena, S. and Islam, A., "Weibull Geometric Process Model for the Analysis of Accelerated Life Testing with Complete Data", International Journal of Statistics and Applications, vol. 2, no. 5, (2012), pp. 60-66, available online: http://dx.doi.org/ doi:10.5923/j.statistics.20120205.03

[18] Zhou, K., Shi, Y. M. and Sun, T. Y., "Reliability Analysis for Accelerated Life-Test with Progressive Hybrid Censored Data Using Geometric Process, Journal of Physical Sciences, vol. 16, (2012), 133-143, http://www.vidyasagar.ac.in/journal/maths/vol16/JPS-V16-14.pdf 